\title{
Considerations when assessing antagonism in vitro: Why standardizing the agonist concentration matters
}

\author{
Peta A. Neale* and Frederic D. L. Leusch
}

Smart Water Research Centre, School of Environment, Griffith University, Southport QLD 4222, Australia

Re-submitted to: Chemosphere

Date: March 2015

doi:10.1016/j.chemosphere.2015.03.054

\footnotetext{
*Corresponding author: p.neale@griffith.edu.au; Ph: +61 755527733
} 
35 There is increasing recognition of the importance of assessing both agonism and antagonism in parallel for environmental samples. Cell-based in vitro assays have the advantage over receptor binding assay that they are able to differentiate between agonist and antagonist activity, but at present there is no standardized approach to assess antagonism in vitro, and in particular the competing agonist concentration can vary in the literature anywhere from half maximal to maximal effect concentrations. In this study, we investigated the influence of changing agonist concentrations in the estrogen receptor alpha $(\mathrm{ER} \alpha)$, progesterone receptor $(\mathrm{PR})$ and glucocorticoid receptor (GR) assays run in antagonist mode. The antagonistic effect varied by over two orders of magnitude when using the range of agonist concentrations applied in the literature, clearly indicating the need for standardization. By comparing antagonist $\mathrm{EC}_{50}$ values with background agonist concentrations, an $\mathrm{EC}_{80}$ background agonist concentration is recommended when assessing antagonism in vitro to optimise both assay sensitivity and reproducibility.

Keywords: Antagonist, estrogen receptor, glucocorticoid receptor, in vitro, progesterone receptor 49 


\section{Introduction}

Environmental waters, such as wastewater and surface water, can contain countless chemical contaminants with different modes of toxic action. Test batteries of in vitro bioassays focusing on endpoints relevant for both human and environmental health are increasingly applied for water quality monitoring and to assess treatment efficiency (e.g. van der Linden et al., 2008; Escher et al., 2014). For a more accurate assessment of effect, it is important to evaluate agonism and antagonism in parallel for receptor mediated endpoints, such as estrogen and progesterone receptor assays. This is because the presence of antagonists in environmental samples may decrease the agonist response, as demonstrated recently by Ihara et al. (2014) for wastewater. Cell-based bioassays can detect antagonism when run in a so-called "antagonist mode", where a potent competing agonist is added at a constant concentration and the suppression of the agonist effect indicates antagonism (Soto et al., 2006).

At present, there is no standard protocol to measure antagonism, and in particular the agonist concentration added can range from the concentration causing $50 \%$ effect $\left(\mathrm{EC}_{50}\right)$ to the maximal effect $\left(\mathrm{EC}_{100}\right)$ (e.g. van der Linden et al., 2008; Ihara et al., 2014). Further, the applied concentration is often not reported. However, competitive antagonists will compete with agonists for the receptor sites; hence the observed effects are likely to change depending on the concentration of agonists and antagonists in the assay.

In this short communication we investigated the implications of changing agonist concentration in estrogen receptor alpha $(\mathrm{ER} \alpha)$, progesterone receptor $(\mathrm{PR})$ and glucocorticoid receptor $(\mathrm{GR})$ assays and suggest a standardized approach for assessing antagonism using in vitro reporter gene assays.

\section{Materials and Methods}

The study was conducted using GeneBLAzer ${ }^{\circledR}$ ER $\alpha$-UAS-bla, PR-UAS-bla and GR-UAS-bla assays (Life Technologies, Mulgrave, Australia). All assays are based on the HEK 293T cell line. The cells were grown in DMEM with GlutaMAX ${ }^{\mathrm{TM}}$ with $10 \%$ dialysed fetal bovine serum (FBS), while phenol red-free DMEM with $2 \%$ charcoal stripped FBS was used for the assay media. 17 $\beta$ Estradiol and tamoxifen were used as the agonist and antagonist in the ER $\alpha$ assay, respectively, while levonorgestrel and RU486 were used as the agonist and antagonist in the PR assay, respectively. RU486 was also the antagonist in the GR assay, with dexamethasone as the agonist (Leusch et al., 2014). The chemical stocks were prepared in methanol (HPLC grade, Fisher Scientific, Scoresby, Australia) and the maximum solvent concentration in the assay was $0.2 \%$. Standard curves with 1:4 serial dilutions for each reference compound were prepared in phenol red- 
free DMEM media in separate 96 well plates, with the agonists serially diluted across the plate and the antagonists serially diluted down the plate. Fifty microliters from both the agonist and antagonist plates for each assay were mixed together in a separate 96 well plate. The final concentration of the reference compounds was $2.4 \times 10^{-14}$ to $2.5 \times 10^{-8}(\log -13.6$ to -7.6$) \mathrm{M}$ for $17 \beta$ estradiol, $1.2 \times 10^{-8}$ to $1.3 \times 10^{-5}$ ( $\log -7.9$ to -4.9$) \mathrm{M}$ for tamoxifen, $9.5 \times 10^{-14}$ to $1.0 \times 10^{-7}(\log -13.0$ to -7.0) $\mathrm{M}$ for levonorgestrel, $2.5 \times 10^{-13}$ to $2.6 \times 10^{-7}(\log -12.6$ to -6.6$) \mathrm{M}$ for dexamethasone and $3.9 \times 10^{-10}$ to $1.0 \times 10^{-7}(\log -9.4$ to -7.0$) \mathrm{M}$ for RU486 (same concentration range in both PR and GR assays). The cells were seeded in black clear bottom 384 well plates with densities of 20,000 cells per well for ER $\alpha$ and GR and 15,000 cells per well for PR. Eight microliters of sample was added to the cells in duplicate and incubated overnight for $16 \mathrm{~h}$ at $37{ }^{\circ} \mathrm{C}$ in a $5 \% \mathrm{CO} 2$ incubator. Standard curves of the agonist and antagonist (with $\mathrm{EC}_{80}$ agonist constant concentration) were included on each plate for assay validation, along with a methanol standard curve with a maximum final solvent concentration of $0.2 \%$, which was added to cells to ensure that the solvent itself was not having an effect. The next day $8 \mu \mathrm{L}$ of LiveBLAzer ${ }^{\text {TM}}$-FRET B/G substrate mixture was added to each well and the plate was incubated at room temperature for $2 \mathrm{~h}$. Fluorescence at 460 and $520 \mathrm{~nm}$ was measured using a Fluostar Omega microplate reader (BMG Labtech, Ortenberg, Germany). The results were expressed as $\%$ maximum response relative to the reference agonist compound.

\section{Results and Discussion}

All studied assays demonstrated that changing the concentration of either the antagonist (in classical agonist mode) or the competing agonist (in antagonist mode) altered the dose-response curves.

\subsection{Impact of the presence of an antagonist on a classical "agonist mode" assay}

As the antagonist concentration increased the agonist curves shifted to the right, increasing the agonist $\mathrm{EC}_{50}$ value and consequently decreasing effect (Figure 1). Within the studied antagonist range, the agonist $\mathrm{EC}_{50}$ values increased by over two orders of magnitude for the $\mathrm{ER} \alpha$ and PR assays and over one order of magnitude for the GR assay. A reduction in agonistic activity in the presence of antagonists has also been previously reported by Ihara et al. (2014) in both human and medaka ER $\alpha$ assays with 17 $\beta$-estradiol and 4-hydroxy-tamoxifen. Further, Barkhem et al. (1998) also observed a concentration-dependent shift in 17 $\beta$-estradiol binding to a human ER $\alpha$ and ER $\beta$ reporter assays in the presence of antagonists ICI 164384 and raloxifene. The observed result was not unexpected as tamoxifen and RU486 are both competitive antagonists. While the percent maximum response dropped to zero in the PR and GR assays, up to $20 \%$ effect was observed with increasing tamoxifen concentrations in the ER $\alpha$ assay. This is not surprising: as well as being an antagonist, tamoxifen is known to be a weak ER $\alpha$ agonist in vitro (Gutendorf and Westendorf, 
121 2001). While tamoxifen is a widely used anti-estrogen in in vitro assays, it may not be the most 122 suitable reference antagonist and other alternatives, such as fulvestrant (ICI 182, 780) (Wilson et al., 2004), may be more appropriate.

\subsection{Impact of the concentration of agonist on an assay run in "antagonist mode"}

126 When operated in antagonist mode, different competing agonist concentrations resulted in a shift in

127 the reported antagonistic effect (Figure 2). As previously stated, competing agonist concentrations 128 range from $\mathrm{EC}_{50}$ to $\mathrm{EC}_{100}$ in the literature, but this can translate into over a 100 fold difference in 129 antagonist $\mathrm{EC}_{50}$ value in the same assay. For example, the $\mathrm{RU} 486 \mathrm{EC}_{50}$ values decreased by 130 approximately two orders of magnitude in the PR assay (log -6.9 to $-8.9 \mathrm{M})$ and the GR assay (- $\log$ 1316.9 to $-8.8 \mathrm{M}$ ) with decreasing constant agonist concentrations. In the $\mathrm{ER} \alpha$ assay the $\mathrm{EC}_{50}$ value for tamoxifen ranged from $\log -6.4$ to $-4.9 \mathrm{M}$ with agonist concentrations from $\mathrm{EC}_{50}$ to $\mathrm{EC}_{100}$. This will obviously hamper comparisons of potency between laboratories that utilise different competing agonist concentrations. In all assays, the antagonists only caused minimal reduction of response at the highest agonist concentration tested, which cautions against the use of excessive agonist background concentrations.

The results clearly show that selecting a lower agonist concentration (e.g., $\left.\mathrm{EC}_{50}\right)$ increases the sensitive of the assay to antagonist compounds. This is an advantage for assessing environmental samples, where compounds may only be present at trace concentrations. However, the downside is that the $\mathrm{EC}_{50}$ is the area of the concentration-effect curve that is most influenced by a minimal change in agonist concentration. This is demonstrated in Figure 3, where the rate of change of the response $(\Delta y)$ as a function of concentration $(\Delta x)$ is greatest at the $\mathrm{EC}_{50}$ concentration. In practical terms, this means that small errors in dosing will have a 1.6 times stronger effect on the variability of the results at $\mathrm{EC}_{50}$ compared to $\mathrm{EC}_{80}$ (based on the rate of change at the two agonist concentrations, Figure 3).

To find the optimal agonist concentration for assessing antagonism in reporter gene assays, a balance has to be found between assay sensitivity and reproducibility. The antagonist $\mathrm{EC}_{50}$ values were compared with the background agonist concentration for each assay, and plotted alongside $\Delta y / \Delta x$ (Figure 3). In all assays, the antagonist $\mathrm{EC}_{50}$ value decreased with decreasing agonist concentration; however, there was little difference in sensitivity at the $\mathrm{EC}_{50}$ and $\mathrm{EC}_{80}$ agonist concentrations. Given that the $\mathrm{EC}_{80}$ concentration is less susceptible to variability compared to $\mathrm{EC}_{50}$, we recommend the use of $\mathrm{EC}_{80}$ background agonist concentration when assessing antagonism in vitro. 
157 A standardization of the competing agonist concentration to $\mathrm{EC}_{80}$ in antagonist mode will allow 158 better comparison between laboratories and hopefully reduce the variability in $\mathrm{EC}_{50}$ values reported for antagonists in the literature.

\section{Acknowledgements}

162 The authors wish to thank Beate Escher (Helmholtz Centre for Environmental Research - UFZ) for 163 valuable input.

\section{References}

Barkhem, T., Carlsson, B., Nilsson, Y., Enmark, E., Gustafsson, J.A., Nilsson, S., 1998. Differential response of estrogen receptor alpha and estrogen receptor beta to partial estrogen agonists/antagonists. Mol. Pharmacol. 54, 105-112.

Escher, B.I., Allinson, M., Altenburger, R., Bain, P.A., Balaguer, P., Busch, W., Crago, J., Denslow, N.D., Dopp, E., Hilscherova, K., Humpage, A.R., Kumar, A., Grimaldi, M., Jayasinghe, B.S., Jarosova, B., Jia, A., Makarov, S., Maruya, K.A., Medvedev, A., Mehinto, A.C., Mendez, J.E., Poulsen, A., Prochazka, E., Richard, J., Schifferli, A., Schlenk, D., Scholz, S., Shiraishi, F., Snyder, S., Su, G., Tang, J.Y.M., Burg, B.v.d., Linden, S.C.v.d., Werner, I., Westerheide, S.D., Wong, C.K.C., Yang, M., Yeung, B.H.Y., Zhang, X., Leusch, F.D.L., 2014. Benchmarking organic micropollutants in wastewater, recycled water and drinking water with in vitro bioassays. Environ. Sci. Technol. 48, 1940-1956.

Gutendorf, B., Westendorf, J., 2001. Comparison of an array of in vitro assays for the assessment of the estrogenic potential of natural and synthetic estrogens, phytoestrogens and xenoestrogens. Toxicology 166, 79-89. Miyagawa, S., Iguchi, T., Tanaka, H., 2014. Co-occurrence of estrogenic and antiestrogenic activities in wastewater: Quantitative evaluation of balance by in vitro ER $\alpha$ reporter gene assay and chemical analysis. Environ. Sci. Technol. 48, 6366-6373. Humpage, A., 2014. Assessment of the application of bioanalytical tools as surrogate measure of chemical contaminants in recycled water. Water Res. 49, 300-315. 
187 Soto, A.M., Maffini, M.V., Schaeberle, C.M., Sonnenschein, C., 2006. Strengths and weaknesses of 188 in vitro assays for estrogenic and androgenic activity. Best Pract. Res. Clin. Endocrinol. Metab. 20, $189 \quad 15-33$.

190 van der Linden, S.C., Heringa, M.B., Man, H.-Y., Sonneveld, E., Puijker, L.M., Brouwer, A., van 191 der Burg, B., 2008. Detection of multiple hormonal activities in wastewater effluents and surface 192 water, using a panel of steroid receptor CALUX bioassays. Environ. Sci. Technol. 42, 5814-5820.

193 Wilson, V.S., Bobseine, K., Gray, L.E., 2004. Development and characterization of a cell line that 194 stably expresses an estrogen-responsive luciferase reporter for the detection of estrogen receptor 195 agonist and antagonists. Toxicol. Sci. 81, 69-77.

196 


\section{Figure Captions}

198

199 Figure 1: Changes in agonist standard curve in the presence of different antagonist concentrations 200 for A) ER $\alpha$ assay with 17 -estradiol (E2) and tamoxifen (TMX), B) PR assay with levonorgestrel 201 (LVG) and RU486 and C) GR assay with dexamethasone (DXM) and RU486 (all concentrations 202 presented as log units).

204 Figure 2: Changes in antagonist standard curve in the presence of different agonist concentrations 205 for A) ER $\alpha$ assay with tamoxifen (TMX) and 17ß-estradiol (E2), B) PR assay with RU486 and 206 levonorgestrel (LVG), and C) GR assay with RU486 and dexamethasone (DXM) (all concentrations 207 presented as log units).

208

209 Figure 3: Changing antagonist $\mathrm{EC}_{50}$ values at different agonist concentrations (left axis, symbols) 210 and derivation $(\Delta y / \Delta x$, blue line) of the agonist dose-response curve (right axis) for A) ER $\alpha$ assay 211 with tamoxifen (TMX) and 17ß-estradiol (E2), B) PR assay with RU486 and levonorgestrel (LVG), 212 and C) GR assay with RU486 and dexamethasone (DXM). The agonist $\mathrm{EC}_{50}, \mathrm{EC}_{80}$ and $\mathrm{EC}_{99}$ 213 concentrations are shown as dashed lines (all concentrations presented as log units). 
$215 \quad$ Figure 1
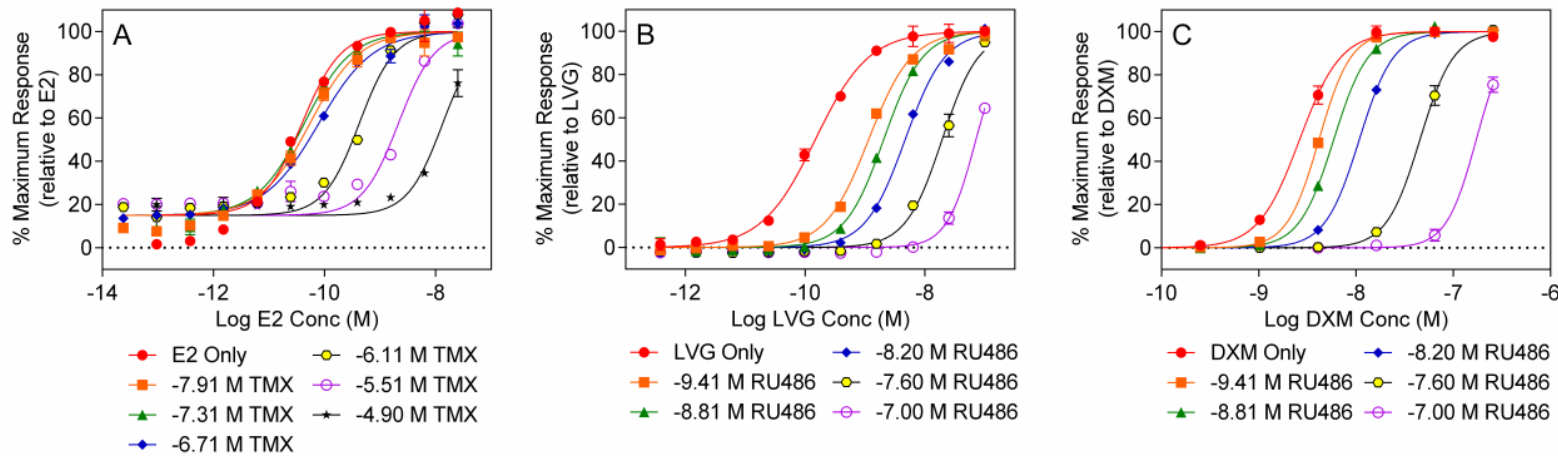
Figure 2
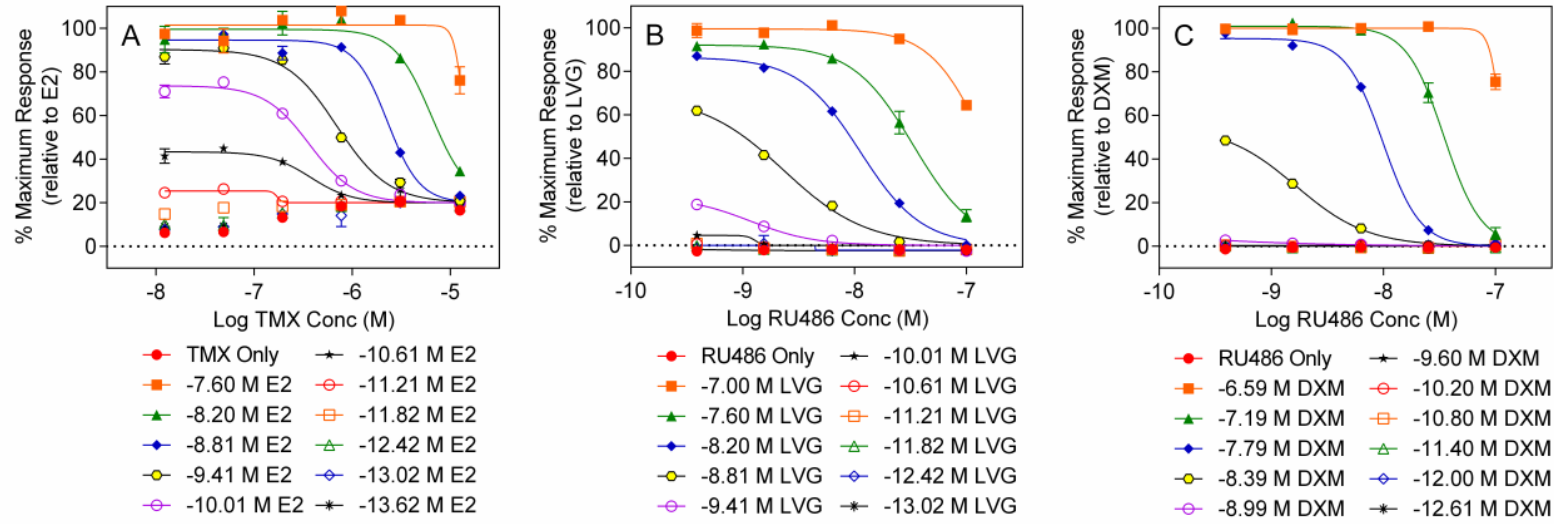
$221 \quad$ Figure 3
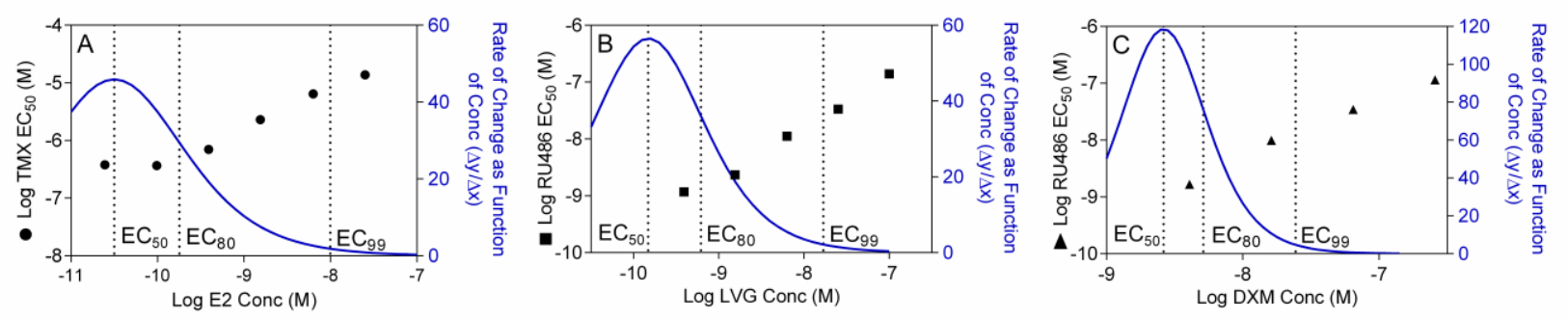